Bernard COMBETTES

Université de Nancy 2 \& UMR-ATILF

\title{
CENTRE ET PÉRIPHÉRIE DANS LE CADRE DE LA GRAMMATICALISATION : LA SPÉCIALISATION DES CATÉGORIES MORPHOSYNTAXIQUES
}

Il peut sembler assez naturel de mettre en relation la notion de marge, de périphérie, et la problématique du changement. L'évolution du système linguistique se caractérise en effet, en grande partie, par l'intégration ou par l'élimination d'unités dont le statut n'est pas central et qui, par là-même, apparaissent comme instables, fortement soumises à la tendance à la simplification et à la régularisation, tours qui constituent des sortes d'exceptions par rapport au fonctionnement général de l'ensemble. Nous nous intéresserons ici à l'évolution elle-même, en essayant de déterminer, en ce qui concerne le cas particulier du français, comment sont hiérarchisés les facteurs qui conduisent à la création de ces éléments périphériques. La constitution progressive d'une classe morphosyntaxique donnée s'opère en effet rarement de façon homogène, certaines unités présentant des caractéristiques spécifiques qui les isolent en partie et leur attribuent une position marginale dans la catégorie. Cette question de la plus ou moins grande intégration d'un élément à une classe doit être rattachée, nous semble-t-il, à la problématique de la grammaticalisation, dont le développement a engendré ces dernières années un renouveau certain dans le champ des études diachroniques. Il convient de prendre ici le concept de grammaticalisation dans son acception large, qui dépasse la définition classique - une expression évolue du domaine lexical au domaine grammatical - pour englober également les cas de changement à l'intérieur du niveau grammatical, en prenant en compte le passage $\mathrm{du}$ «moins grammatical» au «plus grammatical»; il s'agit bien alors de la création de catégories ou de sous-catégories nouvelles, pour lesquelles se pose la question des unités marginales et de leur formation. Les opérations définitoires que sont la réanalyse et l'analogie constituent le principe même qui sous-tend les changements relevant de ce type d'évolution : une expression linguistique ou une structure syntaxique se trouvent réinterprétées, dans des contextes favorables à cette modification, au plan syntaxique comme au plan du contenu, par un ensemble de locuteurs, la nouvelle «analyse » étant ensuite transférée, par une opération proche des procédés métaphoriques, dans d'autres contextes linguistiques. On remarquera au passage que, s'agissant d'états de langue anciens, l'étape de réanalyse ne peut être perçue qu'à travers le résultat de cette généralisation, que dans l'extension analogique. Le point qui nous intéresse ici est donc suggéré par la question suivante : en quoi ces opérations fondamentales et indissociables ont-elles un rapport avec la création d'un centre et d'une périphérie lorsque le processus de grammaticalisation conduit à la formation de catégories nouvelles?

Dans une telle optique, il nous semble possible de formuler une hypothèse, dont nous vérifierons la pertinence par l'analyse de cas particuliers, hypothèse qui pourrait être formulée ainsi : plutôt que de considérer que certaines parties, ou sous-parties, du système linguistique constituent un centre, alors que d'autres, 
moins « régulières », correspondent à la périphérie, on prendra en compte, dans la mesure où il s'agit de diachronie, les tendances de l'évolution, les facteurs qui entrainent le changement. Il conviendra alors de déterminer lesquelles de ces tendances sont centrales, au sens où elles s'appliquent à toutes les unités du domaine concerné, d'autres venant au contraire freiner, limiter, le mouvement général, créant ainsi ce qui apparaîtra comme une irrégularité du système. Les notions de centre et de périphérie ne se trouvent plus appliquées à des faits de langue particuliers, à des composantes du système, mais aux facteurs du changement. Si certaines catégories apparaissent, à travers leurs propriétés, comme centrales ou comme marginales, on peut supposer que ce statut est le résultat du statut - central ou marginal - des règles et des tendances qui ont conduit à leur formation.

\section{La spécialisation des catégories morphosyntaxiques}

Pour illustrer ce jeu des facteurs du changement, ce conflit qui s'établit entre divers paramètres, nous avons choisi deux exemples qui relèvent en fait d'une même problématique: l'évolution et la constitution des classes morphosyntaxiques. On constate en effet que l'un des points importants qui différencie le français du latin réside dans ce qu'il est possible d'interpréter comme une plus ou moins grande spécialisation des catégories et des sous-catégories grammaticales. Cette différence entre les deux systèmes linguistiques se laisse bien percevoir dans le cas de distinctions qui s'établissent entre les pronoms et les déterminants nominaux, ou entre les prépositions et les adverbes, par exemple. Cette tendance à la spécialisation ne peut être séparée d'autres mouvements de fond, dont elle n'est sans doute en fait qu'une des conséquences. On pense en particulier à la profonde restructuration des syntagmes qui accompagne la disparition du marquage des fonctions par un système de déclinaisons. La structure syntaxique "plate», caractéristique du latin classique, laisse la place à une hiérarchisation plus forte de l'énoncé, ce qui entraine la création de classes fonctionnelles comme celle des déterminants, et, plus généralement, la distinction des diverses sous-catégories. On notera que les autres langues romanes n'ont pas poussé aussi loin que le français cette tendance à généraliser l'univocité des formes grammaticales. L'espagnol, par exemple, présente des séries de démonstratifs dont les représentants fonctionnent comme déterminants ou comme pronoms (aquellos libros: ces livres / aquéllos me gustan: ceux-là me plaisent), identiques en cela à des formes latines comme ille ou iste; il en irait de même pour un indéfini comme algunos (algunos libros: quelques livres / algunos me gustan: quelques-uns me plaisent). Sur ce point comme sur d'autres, le système linguistique du français occupe bien une place à part dans l'ensemble des langues de la Romania.

Une première constatation s'impose : une telle modification ne se réalise pas à la même vitesse, au même rythme, pour toutes les catégories. Les démonstratifs, par exemple, trouvent leur équilibre en français préclassique et classique, les formes en cel-constituant le paradigme du pronom, alors que les formes en cetsont réservées aux déterminants. Si l'on excepte quelques tours archaïsants, comme l'emploi des formes de type mien dans : un mien ami, on peut considérer que le microsystème des «possessifs»- la répartition mon / mien - est mis en 
place dès la fin du moyen français. Quant aux indéfinis, ils occupent une place à part, dans la mesure où cette catégorie peu homogène offre l'exemple de traitements très divers. Alors que l'opposition entre deux séries de formes est bien attestée pour quelques / quelques-uns ou chaque / chacun, des unités comme aucun ou plusieurs sont indifférenciées, présentant ainsi un fonctionnement identique à celui de leurs correspondants dans les autres langues romanes. Se posent ainsi des questions auxquelles nous allons tenter de donner un début de réponse: pourquoi certaines régularités s'installent-elles plus rapidement que d'autres, créant ainsi un centre stable ? Dans le système de la détermination nominale, quels sont les facteurs qui ont conduit, pour les indéfinis, à ce que l'on peut considérer comme un statut marginal, périphérique, dans la mesure où il ne présente pas l'homogénéité qui a été généralisée dans les autres composantes ?

Avant d'examiner cette situation particulière de la famille des indéfinis, nous rappellerons rapidement comment s'est opérée et stabilisée la répartition déterminant / pronom pour les autres éléments de la détermination nominale que sont l'article, le «possessif» et le démonstratif. En ce qui concerne l'article, on peut sans doute percevoir une trace de son origine pronominale dans des constructions, limitées à l'ancien français, où les formes de la série le sont utilisées comme anaphores devant un complément non introduit par une préposition :

\section{1 - il abati ton cheval et le Perceval ensemble \\ (il a abattu ton cheval et celui de Perceval) \\ (Queste du Graal)}

2 - celles javelles de mon frere, et les mon pere et les ma mere

$$
\text { (Bible de Macé de la Charité) }
$$

(ces gerbes de mon frère et celles de mon père et celles de ma mère)

Il est difficile de déterminer avec certitude si le est à rattacher à la série pronominale ou s'il s'agit d'un tour elliptique (le cheval de ...) et on peut voir là une zone floue, qui en tout état de cause, va cesser à partir du moyen français. La situation est un peu plus complexe dans le cas du «possessif», qui offre, en français moderne, deux paradigmes, celui de mon, déterminant nominal, et celui de mien, réservé aux emplois pronominaux (le mien) et, dans un registre soutenu, à la fonction d'attribut (ceci est mien). Comme le montrent les exemples suivants, cités par BURIDANT (2000 : 155), cette répartition n'est pas encore clairement établie en ancien français. D'une part, en effet, la série mon peut se rencontrer en combinaison avec un autre déterminant, article ou démonstratif :

3 - apelet Gemalfin, un sun drut (Chanson de Roland)

(il appelle Gemalfin, un son proche $=$ un de ses proches)

4 - par un son fiz a il trop grant dehait

$$
\text { (Poème anglo-normand sur l'Ancien Testament) }
$$

(à cause d'un son fils = un de ses fils, il a un très grand chagrin) 
5 - ces tes serfs qui od mei sunt (Livres des Rois)

(ces tes serfs (= ces serfs qui sont les tiens) qui sont avec moi)

les formes toniques, d'autre part, pouvant fonctionner comme seuls déterminants du nom:

6 - [...] ama jadis une dame, moie sereur (Queste du Graal)

(il aima jadis une dame, mienne sœur)

construction qui donne lieu à un certain nombre de syntagmes prépositionnels à valeur d'adverbiaux, expressions plus ou moins figées, comme : pur tue amor, en moie foi, de moie part (pour tienne amour, en mienne foi, de mienne part). La régularisation s'opère ainsi en deux étapes : le moyen français généralise l'emploi de mien avec d'autres déterminants, accordant à cette forme un fonctionnement d'adjectif, alors que le français moderne limite, si l'on peut dire, la forme tonique aux emplois pronominaux (le mien) et à la fonction d'attribut. Nous ne nous attarderons pas ici sur l'évolution des démonstratifs, nous contentant de renvoyer aux travaux de C. MARChEllo NIZIA (1995; 1999; 2006), qui décrivent comment, dans une longue évolution qui trouve son aboutissement en français préclassique, la série cet- se constitue en déterminant, alors que les formes de type cel-recouvrent la distribution pronominale.

D'une façon générale, on peut considérer que c'est la structuration progressive des syntagmes, en l'occurrence ici du syntagme nominal, hiérarchisation que nous avons évoquée plus haut, qui conduit au développement d'une catégorie spécifique de déterminants, peu à peu distincte de celle des pronoms. Reste toutefois le cas particulier des indéfinis, qui sont loin de présenter la même régularité que les autres sous-catégories et qui se trouvent ainsi dotés d'un statut marginal dans l'ensemble du système de la détermination nominale.

\section{Deux indéfinis : quelque(s) et plusieurs}

Dans une tentative d'explication de l'origine de ce statut, nous invoquerons deux facteurs d'ordre différent. L'exemple de plusieurs et de quelque(s) nous semble bien illustrer ce jeu de divers paramètres. On insistera d'abord sur l'origine de ces formes et sur le déroulement des opérations de réanalyse qui ont conduit des valeurs de départ aux emplois modernes. Dans les deux cas, il est possible de considérer qu'il s'agit d'un processus de grammaticalisation, du moins si l'on accepte, pour cette notion, qu'elle consiste en un mouvement qui conduit du grammatical au "plus grammatical". Ce point commun ne doit cependant pas cacher le fait que cette évolution s'opère dans un mouvement que l'on pourrait en quelque sorte qualifier d'inverse. L'indéfini quelque(s) est fondamentalement, de par sa formation même, de par son étymologie, un déterminant. Issu d'une corrélation de type : quel $N$ que..., qui a la valeur de : un $N$, quel qu'il soit (cf. FOULET, 1919; COMBETTES, 2004), quelque se trouve toujours à proximité immédiate d'un élément nominal, le système corrélatif imposant la présence d'un substantif, comme dans : 
7 - an quel leu que il fust, / Secorre et eidier li deüst

(Chrestien de Troyes, Lancelot 1179)

(en quelque lieu qu'il soit, il devrait le secourir et lui venir en aide)

séquence qui a ensuite évolué, pour des raisons peu claires (cf. FOULET, 1919), vers un redoublement de la forme que, comme dans :

8 - quel que chaperon qu'il portast,

(Jean de Meun, Le roman de la rose, 1273)

(quel que soit le chaperon qu'il portât)

pour aboutir au déterminant quelque, qui conserve, du moins jusqu'au moyen français, son sémantisme de non spécificité (cf. COMBETTES, 2004). Quelles que soient les raisons qui ont pu favoriser le figement de la locution, il ne semble guère possible de voir l'origine du déterminant quelque dans une forme qui serait fondamentalement pronominale, ce qui est le cas de plusieurs, comme nous le verrons. C'est ce point de départ qui explique, nous semble-t-il, la différence qui sépare plusieurs de quelques-uns; alors que plusieurs pouvait se juxtaposer à un syntagme nominal et prendre peu à peu le statut de déterminant en passant par celui d'adjectif, un déterminant du type de quelque n'est pas l'objet d'une grammaticalisation qui serait, à l'inverse, un mouvement de détachement et d'autonomisation. Tout se passe comme si quelque, fondamentalement attaché à la fonction de déterminant, ne pouvait, à lui seul, constituer une unité dotée d'un fonctionnement de syntagme nominal, mais devait être accompagné d'un support, en l'occurrence un(s), correspondant à la tête du groupe. L'opération de réanalyse peut être perçue dans des énoncés qui présentent une ambiguité, au plan syntaxique et au plan sémantique, comme dans l'exemple :

9 - vous devez confesser a vostre curé du moins a quelque ung prestre ou religieux ayant sa puissance (Les cent nouvelles nouvelles, 1456)

où un peut être rattaché à quelque, le substantif prêtre jouant un rôle d'apposition, ou être interprété comme déterminant du groupe un prêtre.

Nous ajouterons que le figement de quelqu'un, ainsi que le passage, d'un point de vue sémantique, du non spécifique au spécifique, ont pu être favorisés par des tours «partitifs», que l'ensemble de référence soit construit en fonction de complément prépositionnel :

10 - survint quelque ung des serviteurs du chasteau (Le Clerc, 1502)

ou qu'il soit évoqué dans le contexte, l'indéfini fonctionnant alors pleinement, avec sa valeur première, comme anaphorique :

11 - et ses serviteurs qui s' en mesloient estoient peu experimentéz et parresseux ; et croy que quelque ung avoit intelligence avecques le pape

(Commynes, Mémoires t. 3, 1489) 
L'aboutissement de cette évolution est la disparition de toute mention d'un ensemble de référence, ce qui permet à quelqu'un de renvoyer à l'indéfinition la plus large dans la catégorie des animés (toute personne, quelle qu'elle soit), valeur qui sera la seule à se maintenir après la période du moyen français.

On voit donc comment l'origine du tour, le point de départ du processus de grammaticalisation, conditionne, en quelque sorte, le type d'évolution et conduit à des réalisations différentes d'un même mouvement de fond. Il n'en va pas de même en effet pour l'indéfini plusieurs, qui, s'il peut apparaître dès l'origine en fonction de déterminant, ou, du moins, en position d'adjoint au nom, n'en a pas moins une valeur fondamentale d'anaphorique. L'étymologie couramment admise pour cet indéfini est celle de *plusiores, comparatif formé sur plus ; cette dernière forme, dans un mouvement assez fréquemment représenté dans les langues, est l'objet, dès le latin, d'un glissement de la valeur comparative de «plus » vers la valeur intensive de «beaucoup, très ». C'est alors *plusiores qui occupe la place qui était occupée par plus. L'expression du comparatif, caractéristique première de plusieurs, se maintient encore nettement en ancien français, en particulier dans des corrélations avec que, comme dans les passages suivant, que l'indéfini fonctionne en tant qu'adjectif attribut ou en tant que nominal :

12 - Franceis furent plusur que cil de Normandie

(Wace, Roman de Rou, XII ${ }^{\text {ème }} \mathrm{s}$.)

(les Français furent plus nombreux que ceux de Normandie)

13 - El regne Deu, u alat il, Par lui en vunt plusur que mil.

(Vie de Saint Brendan, 1129)

(au royaume de Dieu, où il alla, grâce à lui il en va plus que mille)

Il nous semble possible de considérer que c'est cette orientation, liée à l'étymologie même du terme, à partir d'un point de repère qui demeure sousjacente à cet indéfini et qui fait que la relation anaphorique est fondamentale, dans la mesure où l'ensemble de référence est d'ordinaire préalablement évoqué. Dès le $\mathrm{XII}^{\mathrm{ème}}$ siècle, plusieurs semble pouvoir fonctionner comme adjoint à un élément nominal ; c'est du moins ce qu'on peut déduire d'une variante comme :

14 - Malsfeiz veient cent et plusurs

(des méfaits ils voient cent et plus)

15 - Malsfeiz veient millers plusurs

(des méfaits ils voient plusieurs milliers)

qui témoigne du passage de la valeur anaphorique : cent et plus (que cent) à une valeur de détermination de type adjectival : des milliers plus que un.

Le rattachement contextuel inhérent à plusieurs explique le type de grammaticalisation qui affecte cet indéfini ; plusieurs indices vont dans le même sens et permettent d'interpréter le passage à la catégorie déterminant comme un 
processus d'adjonction, de juxtaposition, qui, dans un premier temps, partage la plupart des caractéristiques des adjectifs épithètes. On relèvera aussi que, lorsqu'il est utilisé comme pronom, avec les fonctions habituelles des nominaux, plusieurs est souvent complété par un syntagme prépositionnel introduit par de, plusieurs de $\mathrm{N}$ :

\section{6 - De telz ay je plusieurs congneu qui [...] (Jean Daudin, 1360)}

Cette construction peut sembler normale, mais il est intéressant de noter le fait que cette complémentation, de règle en français moderne, est fortement concurrencée, en moyen français, par la juxtaposition de plusieurs et du groupe nominal, en particulier lorsque celui-ci est doté d'un déterminant possessif :

\section{7 - a l' encontre de plusieurs ses adversaires (Juvénal des Ursins, 1440) \\ 18 - et tint manière de donner congié à plusieurs ses serviteurs}

\section{(La Marche, 1470)}

Devant ce type d'exemples, on peut s'interroger sur la nature exacte de plusieurs : anaphore à valeur nominale, complétée par un syntagme nominal en apposition, ou, déjà, prédéterminant, adjectif antéposé aux éléments nominaux ? L'ambiguïté de telles séquences n'a pu que constituer des contextes favorables à la réanalyse, dans la mesure où c'est la disparition de ces tours qui permettra de considérer que plusieurs entre dans un paradigme de déterminants obligatoires, dans un système d'opposition avec l'article, le démonstratif et le possessif.

On peut ainsi faire l'hypothèse que le pronom plusieurs se fixe peu à peu en position de déterminant, mais il faut également constater que cette grammaticalisation ne va pas jusqu'à l'érosion qu'on pourrait attendre, érosion observable par exemple dans des processus plus anciens, pour le (de illum) ou pour cet (de *ecce iste). Le déterminant quelque, pour sa part, doit s'adjoindre la forme un pour constituer un groupe à fonctionnement de pronom. Le passage du pronom au déterminant ne se réalise pas d'une manière identique à celui du déterminant au pronom. C'est cette différence dans l'évolution qui crée, nous semble-t-il, des unités marginales, en l'occurrence plusieurs et des unités plus intégrées, comme quelques. Si quelques a pu s'intégrer sans difficulté dans un système qui oppose des séries de formes distinctes, c'est que le point de départ de la grammaticalisation de cet indéfini le permettait. On distinguera ainsi des règles générales et des règles plus locales, qui peuvent entrer en conflit, perturbant alors le cours normal de l'évolution.

Si l'origine différente des formes doit être prise en considération, il ne faut pas négliger la chronologie même du changement. On peut par exemple se demander pourquoi une forme comme certains n'est pas entrée, comme quelques, en combinaison avec uns, pour aboutir à *certains-uns. Une explication plausible serait que cet indéfini ne s'est grammaticalisé comme pronom, à partir d'une valeur adjectivale, que très tardivement (le Robert Historique date en effet de 1634 la première attestation, par ailleurs isolée), à un moment où un(s) n'a plus le statut qu'il avait dans les formations anciennes comme li uns de l'ancien français ou 
quelqu'un du moyen français. Le déterminant un n'apparaît plus comme une forme « libre », dotée d'une autonomie qui aurait permis la combinaison avec certains.

Il faut noter au passage qu'une autre tendance a pu intervenir et constituer une nouvelle cause de déséquilibre. En effet, dans le cas de formes comme moult et maint, qui fonctionnent davantage comme adjectifs que comme véritables déterminants, la grammaticalisation n'a pu se réaliser dans la mesure où ces indéfinis ont été éliminés de l'usage courant par des périphrases comme beaucoup de et ses variantes (un tas de, une foule de, pas mal de, etc.). On peut voir là une conséquence du mouvement général, bien représenté dans l'ensemble du système, vers les constructions analytiques, mouvement qui aboutit, dans ce cas particulier, à l'explicitation de la préposition de, sous-jacente aux indéfinis quantitatifs.

Nous avons évoqué jusqu'à présent, qu'il s'agisse de l'étymologie ou de la datation de la grammaticalisation, l'influence de facteurs qui relèvent de la dimension chronologique ; c'est dans la diachronie et dans la réalisation des divers modes de changement qu'il faut rechercher les raisons de la constitution d'un centre et d'une périphérie.

D'un point de vue plus synchronique, il convient également de prendre en compte la spécificité sémantique des indéfinis à l'intérieur de la classe des déterminants. Les informations données par des formes comme quelques ou plusieurs sont d'un autre ordre que celles qui sont portées par les déterminants spécifiques ; ces derniers ne peuvent se combiner entre eux (*le ce, *ce mon, etc.) et renvoient aux concepts de base de la définitude et de la deixis. Les indications fournies par les indéfinis, qu'elles relèvent du domaine quantitatif ou de l'expression de l'identité, peuvent être considérées comme subordonnées à ces traits fondamentaux. Cette hiérarchisation des traits sémantiques entraîne pour les indéfinis, du moins pour certains d'entre eux, un statut intermédiaire entre celui des déterminants et celui des adjectifs. Le fonctionnement de cette zone floue se laisse bien observer dans le cas des marqueurs d'identité ou de différence comme même, tel, pareil, autre, formes qui, jusqu'à l'époque classique, ne sont pas obligatoirement accompagnées d'un déterminant spécifique, ce dont on peut voir un vestige dans des expressions comme autre part ou de même sorte, par exemple. Ici encore, c'est un facteur particulier, relevant du domaine sémantique, qui contribue à l'isolement d'une sous-classe en empêchant ce qui correspondrait à une évolution «normale », qui serait la réalisation de la tendance générale à distinguer les catégories.

\section{L'alternance que / ce que dans les locutions conjonctives}

Un deuxième exemple nous permettra de mieux cerner les facteurs d'ordre divers qui viennent troubler la régularité des évolutions et conduisent ainsi à des déséquilibres, à la création d'unités périphériques moins bien intégrées dans l'ensemble du système. Il s'git de la répartition entre la forme simple que et l'expression ce que dans les locutions conjonctives comme: pour que / parce que. On peut, ici encore, parler de grammaticalisation, dans la mesure où, même s'il n'y a pas vraiment passage du grammatical au «plus grammatical », la création de ces tours correspond bien à un changement de catégorie comportant une étape de réanalyse qui débouche sur la formation de locutions. Du point de vue 
diachronique, cette alternance que / ce que est le résultat de l'origine différente des deux marqueurs de subordination. Nous sommes en effet en présence de deux types d'hypotaxe, que présente déjà le système du latin, qui distingue des subordonnants formés sur des corrélations du type comparatif, combinant un adverbial et quam (ante ... quam; post ... quam) et des locutions où, sur le modèle de la subordination relative, quod est précédé d'un anaphorique (pro eo quod: pour que...). On assiste, en français, comme dans le cas des déterminants nominaux, à la distinction progressive de sous-catégories et, ici encore, divers facteurs interviennent et pèsent sur la réalisation de ce mouvement de grammaticalisation. Notons d'abord que l'évolution aurait pu aller vers la formation d'un système où se seraient opposés conjonctions et relatifs; ce n'est pas cette direction qui a été privilégiée, et une séquence comme ce que demeure ambiguë dans certains contextes. Le type de spécialisation a été conditionné par la nature du premier élément de la locution, ce qui montre à nouveau l'importance de l'origine des tours. Les prépositions «pures», qui n'ont pas également un fonctionnement adverbial, ne peuvent en effet entrer dans des corrélations comme avant ... que, et se combinent obligatoirement avec ce, suivant en cela le modèle d'une locution latine comme ad id quod, alors que les formes avant, après, depuis s'insèrent sans difficulté dans le schéma ante ... quam, qui ne nécessite pas la présence du démonstratif. De cette opposition découle la spécialisation des locutions en ce que dans le rôle d'introducteur de subordonnées régies par le verbe principal (on s'attend à ce qu'il vienne; on s'étonne de ce qu'il soit venu) et les locutions en que comme introducteurs de propositions circonstancielles (avant que, depuis que, etc.), répartition qui correspond à la spécificité des prépositions concernées. En fait, l'opposition n'est pas aussi nette, historiquement du moins. L'autonomie syntaxique de la forme ce permettait la construction de nombreux syntagmes prépositionnels compléments, comme : après ce, pour ce, sur ce, etc. et l'influence de ces groupes prépositionnels à fonction de circonstants a conduit à la formation de locutions en ce que et à des variantes comme : avant que / avant ce que ou depuis que / depuis ce que. L'évolution ne va pas jusqu'à la généralisation de ce que, qu'on aurait pu attendre, divers faits venant entrâner des déséquilibres. Intervient en effet ici un facteur d'ordre sémantique, lié au rôle du contexte dans la grammaticalisation: les tours en que apparaissent en effet comme non marqués, dans la mesure où ils peuvent être utilisés dans tous les types d'environnements, en particulier avec des subordonnées dont le contenu renvoie à l'itératif ou à des modalités comme l'éventuel, l'irréel, le souhaitable, alors que les formes en ce que se voient limitées à l'introduction de propositions de l'ordre du réel, de l'asserté. On pourra comparer les emplois de dès (ce) que dans les extraits suivants, de la fin du XV ${ }^{\text {ème }}$ siècle :

19 - Dès ce que ledict duc le sceüt, il fist dire à ceulx qui l' avoient qu' ilz le transportassent sans luy en rien dire

(Commynes)

20 - Et estoit nostre façon de proceder que, dès que nous estions arrivéz au logis dudit duc, il venoit au devant de nous et la duchesse jusques au bout d'une galerie 
21 - Dès que ledict duc fut desarmé, appella ung secretaire et fist escrire unes lettres au connestable

(id.)

Les deux premiers exemples illustrent l'alternance ce que / que en opposant un prédicat qui renvoie à un événement réel et unique (il le sut) et un prédicat à valeur itérative (chaque fois que nous étions arrivés); le troisième exemple témoigne de l'emploi non marqué de que, qui introduit une subordonnée identique à celle de (19). Une répartition du même ordre peut être relevée pour depuis (ce) que :

22 - Item, dist que, depuis ce qu'il a esté delivré de prison de Chastellet par les graces de la royne, il seul a prins et gaigné, d'une vielle merciere qui [...]

(Registre du Châtelet)

23 - je vous dy que, depuis que une puissance tourne le dos devant une autre, qu'elle pert le cuer; et en icellui cas vous ne devés point faindre à les assaillir

(Jean de Bueil)

24 - Chers maistres et grans amis, depuis que vous m'escripsistes, me sont plusieurs aventures avenues

(Froissart)

Alors que le premier de ces passages fait référence à un événement réel et attesté (il a été délivré), le deuxième renvoie à une vérité générale, qui, en français moderne, serait rendue par dès que. On notera d'ailleurs que la locution évolue facilement, dans ce cas, vers une valeur qui dépasse la simple temporalité, la relation des deux propositions correspondant à l'expression d'une condition nécessaire et suffisante : il suffit que $\mathrm{P} 1$ pour que $\mathrm{P} 2$. Le troisième exemple montre la neutralisation qui s'effectue avec que, utilisé dans le contexte d'un événement unique et réalisé.

Dès le moyen français, les locutions en que prennent ainsi le pas sur leurs variantes en ce que, et constituent le centre du système, les formes en ce que apparaissant comme périphériques et réservées à des contextes syntaxiques bien déterminés.

Reste toutefois le cas de parce que, qui semble isolé dans l'ensemble du système, comparé par exemple à la locution pour que, qui a, de façon attendue, supplanté pour ce que. On pourrait certes considérer que c'est la nature de par, préposition «pure » et non adverbe, qui impose la présence du démonstratif, la séquence * par que étant sentie comme agrammaticale au même titre que *à que ou *de que. Il n'en reste pas moins que des formes comme dès, pour, ou sans sont entrées dans le jeu de l'opposition subordonnée essentielle / subordonnée circonstancielle, avec l'élimination de dès ce que, pour ce que, sans ce que. La marginalité de parce que s'explique peut-être par l'influence des contextes dans lesquels la subordonnée est à interpréter comme un «complément d'agent » et non comme un circonstanciel, un énoncé comme : je suis étonné par ce qu'il est venu correspondant à : je suis étonné par le fait qu'il est venu. Ce type d'emploi, sans 
doute à l'origine de la formation de la locution (la subordination causale étant par ailleurs exprimée par pour ce que), fait en quelque sorte basculer parce que du côté de à ce que et de ce que, introducteurs de constituant essentiel, alors que les subordonnées introduites par des locutions comme pour que, sans que ou dès que correspondent pleinement à la fonction de circonstant.

Par l'examen de ces deux cas particuliers, nous avons essayé de montrer que la répartition des unités en éléments centraux et en éléments marginaux pouvait être interprétée comme le résultat, plus ou moins direct, d'une hiérarchisation des facteurs, qui entrent en jeu dans le processus du changement, tant en ce qui concerne l'origine de l'innovation que le mode de l'évolution. La tendance à spécialiser les catégories morphosyntaxiques serait ainsi une tendance centrale qui, si elle n'était pas freinée par d'autres causes, conduirait à un système en équilibre, dans lequel la zone périphérique serait fortement réduite ; il en irait de même pour le passage du modèle synthétique au modèle analytique. Seraient alors à classer comme périphériques des contraintes plus locales, qui ne s'appliqueraient qu'à des cas particuliers et qui appartiendraient davantage au plan synchronique : la nature des unités soumises au changement, le sémantisme spécifique de certaines d'entre elles, relèveraient ainsi de cette deuxième catégorie. Dans une telle perspective, les « exceptions », les tours « hors système », ne sont que la conséquence d'un conflit entre deux types de facteurs, dont les uns peuvent être considérés comme centraux et les autres comme périphériques.

\section{BIBLIOGRAPHIE}

BURIDANT, C. (2000), Grammaire nouvelle de l'ancien français, Paris, Sedes. Combettes, B. (2004), «Quelque : aspects diachroniques », Scolia, 18, p. 9-40.

FOULET, L. (1919), «Quelque », Romania, 45, p. 220-249.

Hengeveld, K., RiJKhoff, J. \& A. SiEWIERSKA (2004), « Parts-of-speech systems and word order », Journal of Linguistics, 40, p. 527-570.

MARCHEllo-Nizia, C. (1995), L'évolution du français, Paris, Armand Colin.

MARCHEllo-NizIA, C. (1999), Le français en diachronie, Paris, Ophrys.

MARCHELlO-NIZIA, C. (2006), Grammaticalisation et changement linguistique, Bruxelles, De Boeck.

MEILlET, A. \& J. VENDRYES (1960), Traité de grammaire comparée des langues classiques, Paris, Champion.

SCHNEDECKER, C. (à par.), «Certain et ses avatars : approche diachronique », Scolia.

VINCENT, N. (1997), « The emergence of the D-system in Romance. », in : A. van Kemenade \& N. Vincent, (éds.), Parameters of Morphosyntactic Change, Cambridge, Cambridge University Press, p. 149-169. 


\section{RESÚMEN}

El objetivo de este trabajo es mostrar cómo los elementos periféricos son el resultado de un conflicto entre dos tipos de reglas de evolución: las principales tendencias de cambio y las otras, más específicas, dentro de los microsistemas. Esta aplicación de las reglas se ilustra con dos ejemplos: en primer lugar se considera el caso de los indefinidos, que son una clase heterogénea en francés. La comparación de "quelques" y "plusieurs" muestra que el origen de las expresiones puede obstaculizar la tendencia general hacia la especialización de las categorías. El segundo ejemplo es la oposición "que" / "ce que" en las locuciones conjuntivas. Se observan los diferentes factores sintácticos y semánticos que han tenido lugar en la constitución del sistema del francés moderno. 